\title{
The profile of lysosomal exoglycosidases in replicative and stress-induced senescence in early passage human fibroblasts
}

\author{
Małgorzata Knaś ${ }^{1}$, Anna Zalewska ${ }^{2}$, Rafał Krętowski ${ }^{3}$, Marek Niczyporuk ${ }^{4}$, \\ Napoleon Waszkiewicz ${ }^{5}$, Marzanna Cechowska-Pasko ${ }^{3}$, Danuta Waszkiel ${ }^{2}$, Krzysztof Zwierz ${ }^{6}$
}

\author{
${ }^{1}$ Research Laboratory of Cosmetology, Medical University of Bialystok, Poland \\ ${ }^{2}$ Department of Pedodontics, Medical University of Bialystok, Poland \\ ${ }^{3}$ Department of Pharmaceutical Biochemistry, Medical University of Bialystok, Poland \\ ${ }^{4}$ Research Laboratory of Esthetic Medicine, Medical University of Bialystok \\ ${ }^{5}$ Department of Psychiatry, Medical University of Bialystok, Poland \\ ${ }^{6}$ Medical College of the Universal Education Society, Lomza, Poland
}

\begin{abstract}
The aim of the present study was to assess the profiles of the exoglycosidases: $\mathrm{N}$-acetyl- $\beta$-hexosoaminidase, $\beta$ glucuronidase and $\beta$ galactosidase, $\alpha$ mannosidase and $\alpha$ fucosidase in fibroblast culture undergoing replicative and stress-induced senescence. Half of the cell culture was grown in normal conditions, without the stressor, and the other half of the cell was treated with $0.15 \mathrm{mM}$ tert-butylhydroperoxide. The activities of total $\mathrm{N}$-acetyl $-\beta$-hexosoaminidase as well as $\beta$ glucuronidase in the cell lysate were determined in duplicates using the method of Marciniak et al. The activities of $\beta$ galactosidase, $\alpha$ mannosidase and $\alpha$ fucosidase in the cell lysate were determined in duplicates using the method of Chatteriee et al. with the modification by Zwierz et al. The activities of the exoglycosidases examined, with the exception of $\beta$ glucuronidase, showed a significant increase between individual days of the experiment in both non-stressed and stressed fibroblast cell culture. On each day of the experiment, in the cell lysate of stressed fibroblasts, the activities of exoglycosidases were significantly higher compared to the non-stressed cells. There were very strong correlations between SA- $\beta$-GAL staining and $\beta$ galactosidase activity on individual days of the experiment in both non-stressed and stressed fibroblast cell culture. Replicative and stress-induced senescence results in significant changes to the level of lysosomal exoglycosidases, and results in enhanced lysosomal degradative capacity. (Folia Histochemica et Cytobiologica 2012, Vol. 50, No. 2, 220-227)
\end{abstract}

Key words: exoglycosidases, fibroblast cell culture, replicative and stress-induced senescence

\section{Introduction}

It was Hayflick and Moorhead [1] who first demonstrated the ageing-associated molecular changes in human cells. They observed that during consecutive

Correspondence address: M. Knaś,

Research Laboratory of Cosmetology,

Medical University of Bialystok,

Akademicka Str. 3, 15-267 Bialystok, Poland;

tel.: + 488574858 20, fax: + 488574858 28;

e-mail:knass@umb.edu.pl cultivation, after settlement of the primary culture, fibroblasts divided at a rather constant rate in mass cultures. After a certain number of divided cycles, an irreversible cessation of the cell growth was observed. This phenomenon was called 'replicative senescence' [2]. It depends on telomere shortening linked to the DNA end-replication problem, overexpression of certain oncogenes or tumor suppressors [3]. Premature senescence, also known as stress-induced senescence, develops after exposure to a diversity of oxidative stresses and DNA-damaging factors [3, 4]. 
The senescent phenotype includes growth arrest, increased cell size and flattening [4], with an increased number and increased activity of lysosomes and SA$\beta$-galactosidase (senescence-associated $\beta$-galactosidase; SA $\beta$-GAL) [5].

Exoglycosidases are involved in post-translational modifications of glycoproteins and the degradation of different glycoconjugates [6]. The first process occurs in the endoplasmic reticulum and Golgi apparatus [6], while the degradation of glycoconjugates is takes place mainly in lysosomes [7] and, to a lesser extent, in proteosomes [8]. Lysosomal exoglycosidases participate in reactions in which the previous reaction product is a substrate for another enzyme. Oligosaccharide chains of glycoconjugates are hydrolyzed at the same time from the non-reducing and reducing ends. Proteinase activity begins to digest the protein core, releasing the oligosaccharide with Asn. Parallel sialidases hydrolyze sialic acid at the non-reducing end of the oligosaccharide. Next, galactosidase and fucosidase release $\alpha$-galactose at the non-reducing end and $\alpha$-fucose at the reducing end, respectively [9]. The next step is hydrolysis of the N-glycosidic bond (between the oligosaccharide and Asn) with the aspartylglucosaminidase participation [10]. The release of Asn permits endo- $\mathrm{N}$-acetylglucosaminidase, which hydrolyzes the bond between $\mathrm{N}$-actetylglucosamine and the polypeptide chain and $\mathrm{N}$-actetylglucosamine of the oligosaccharide chain. $\mathrm{N}$-acetylglucosaminidase hydrolyzes the bond between $\mathrm{N}$-actetylglucosamine at the non-reducing end of the remaining part of the oligosaccharide and a mannose [11]. The final stage in the catabolism of glycoproteins is the mannosidase action to release the rest of the $\alpha$ and $\beta$ mannose at the non-reducing end of the oligosaccharide [12].

To the best of our knowledge, nobody has previously evaluated the relationship between exoglycosidase activities and replicative or stress-induced senescence. Therefore, the aim of the present study was to assess the profiles of the exoglycosidases: $\mathrm{N}$-acetyl- $\beta$ hexosoaminidase (HEX), $\beta$ glucuronidase (GLU) and $\beta$ galactosidase (GAL), $\alpha$ mannosidase (MAN) and $\alpha$ fucosidase (FUC) in fibroblast culture undergoing replicative and stress-induced senescence.

\section{Material and methods}

Cell culture. Normal primary skin fibroblasts (p8) were purchased from ATCC (CRL1474). Fibroblast cultures were passaged in high glucose $(4.5 \mathrm{~g} / \mathrm{L})$ DMEM without L-glutamine with $10 \%$ FBS in six-well plates (Falcon). Cultures were grown at $37^{\circ} \mathrm{C}$ in an atmosphere containing $5 \%$ $\mathrm{CO}_{2}$ For the first two days, cells were grown up to $60 \%$ of confluence, and the third day of the cell culture was the first day of the experiment. Half of the cell culture was submitted to sub-lethal stress under $0.15 \mathrm{mM}$ tert-butylhydroperoxide ( $t$-BHP) (Sigma-Aldrich) [13] during the consecutive five days of the experiment. Cells were treated with $t$-BHP 24 hours before starvation. The other half of the cell culture was grown in normal conditions, without the stressor (Table 1). Every 24 hours, equal numbers of cells were collected, washed three times in PBS, and re-suspended in $200 \mu 1100 \mathrm{mM}$ phosphate-citrate buffer $\mathrm{pH} 4.3$ for GAL, FUC, MAN, and 4.7 for HEX, and in $200 \mu 1100 \mathrm{mM}$ acetate buffer 4.5 for GLU. Cells were sonicated with an ultrasonic cell disrupter three times for 20 seconds on ice. The cell lysates were centrifuged at $12,000 \mathrm{~g}$ for $10 \mathrm{~min}$ at $4^{\circ} \mathrm{C}$. As a substrate to determine the HEX activity, 4-nitrophenyl- N-acetyl- $\beta$-glucosaminide (Sigma, St. Louis, MO, USA) was used, as well as p- nitrophenyl- $\beta$-D-glucuronide (Fluka Chemie; Sigma, St. Louis, MO, USA) for GLU, and p-nitrophenyl- $\beta$-D-galactopyranoside (Sigma) for GAL, p-nitrophenyl- $\alpha$-D-fucopyranoside (Sigma) for FUC, and p-nitrophenyl- $\alpha$-D-mannopyranoside for MAN (Sigma). The activities of total HEX as well as GLU in the cell lysate were determined in duplicates using the method of Marciniak et al. [14]. The activities of GAL, FUC, and MAN in the cell lysate were determined in duplicates using the method of Chatteriee et al. [15] with the modification by Zwierz et al. [9]. Spectrophotometric measurements of p-nitrophenol realased by the exoglycosidases activity were carried out at $405 \mathrm{~nm}$ using a microplate reader MINDRAY MR-96A.

To confirm senescence, cell staining for SA- $\beta$-GAL was performed every 24 hours according to the method of Dimri et al. [16] using the senescence detection kit BioVision (USA) [20] (Table 1). The intensity of staining of the stained fibroblasts was rated by counting the Area Under a Curve (AUC) of the graph obtained in the Gene Tools Imagine Analysis Software.

Statistical analysis. Statistical analyses were done using Statistica v 9.0 (StatSoft, Krakow, Poland) according to ANOVA, post hoc test and $t$-Student test. Pearson's correlation coefficient was used to determine the association between two variables. Results were expressed as means \pm SD. Statistical significance was defined as $\mathrm{p}<0.05$.

\section{Results}

HEX, GAL, and MAN activities showed a significant increase between individual days of the experiment in both non-stressed and stressed fibroblast cell cultures (Figures 1A-C). However, on each day of the experiment, in the cell lysate of stressed fibroblasts, the activities of HEX, GAL and MAN were significantly higher compared to the non-stressed cells (Figures $2 \mathrm{~A}-\mathrm{C}$ ).

The FUC activity in the cell lysate of non-stressed fibroblasts showed a strong tendency to increase be- 
Table 1. Several parameters of cell culture of fibroblasts

\begin{tabular}{|c|c|c|c|c|c|c|c|c|}
\hline \multirow[t]{2}{*}{ Day of experiment } & \multicolumn{2}{|c|}{ Volume of cells ( $p L$ ) } & \multicolumn{2}{|c|}{ Number of cells $($ cell $/ \mathrm{mL})$} & \multicolumn{2}{|c|}{ Size of cells $[\mathrm{mm}]$} & \multicolumn{2}{|c|}{ Photo of staining } \\
\hline & NS & $\mathbf{S}$ & NS & $\mathbf{S}$ & NS & $\mathbf{S}$ & NS & $\mathbf{S}$ \\
\hline $1^{\text {st }}$ & 5.310 & 5.270 & $2.080 \times 10^{5}$ & $2.157 \times 10^{5}$ & 20.25 & 21.58 & & \\
\hline $2^{\text {nd }}$ & 5.310 & 4.998 & $2.310 \times 10^{5}$ & $2.280 \times 10^{5}$ & 20.48 & 20.12 & & \\
\hline $3^{\text {rd }}$ & 5.070 & 6.010 & $2.541 \times 10^{5}$ & $2.419 \times 10^{5}$ & 24.19 & 23.81 & & \\
\hline $4^{\text {th }}$ & 6.453 & 5.941 & $3.012 \times 10^{5}$ & $3.001 \times 10^{5}$ & 21.39 & 22.04 & & \\
\hline $5^{\text {th }}$ & 5.094 & 5.804 & $3.321 \times 10^{5}$ & $3.041 \times 10^{5}$ & 23.00 & 21.90 & & \\
\hline
\end{tabular}

NS - non-stress; S - stress

tween the first and the third day, and a significant increase between the third and the fifth day of the experiment (Figure 1D). The FUC activity in the cell lysate of stressed cells showed a strong tendency to increase between the first and the second day of the experiment, and also showed a significant increase between the second and the fifth day of the experiment (Figure 1D). On the first day of the experiment, a strong tendency to increase was observed in the FUC activity in stressed fibroblasts compared to nonstressed cells. On the following days of cell culture, the FUC activity was significantly higher in the stressed fibroblasts than in the non-stressed cells (Figure 2D).

There was a very weak tendency to increase in the GLU activity between individual days of the experiment in both non-stressed and stressed fibroblast cell cultures (Figure 1E). However, on each day of the experiment, in the cell lysate of the stressed fibroblasts the GLU activity was significantly higher compared to the non-stressed cells (Figure 2E).

There were very strong correlations between SA- $\beta$-GAL staining and GAL activity on individual days of the experiment in both non-stressed and stressed fibroblast cell cultures (Figures 3A-E). There were no correlations between SA- $\beta$-GAL staining and the activities of the remaining exoglycosidases (data not shown).
The data for the GAL activities and SA- $\beta$-GAL staining on individual days of the experiment in both non-stressed and stressed fibroblast cell cultures has been fitted to a $2^{\text {nd }}$-order polynomial regression line with a perfect fit $\left(R^{2}=0.99, R^{2}=0.98, R^{2}=0.97\right.$ and $R^{2}=0.98$, respectively) (Figure 4).

\section{Discussion}

It has been shown that some lysosome activities that are up-regulated in replicative senescent cells, as well as in the cells, undergo oxidative stress, e.g. GAL activity, acid phosphatase, cathepsin B and D $[16,17]$. To the best of our knowledge, this is the first study to examine the activities of lysosomal exoglycosidases: HEX, GLU, MAN, and FUC in cells which undergo replicative and stress-induced senescence. We demonstrated for the first time a strong relationship between SA- $\beta$-GAL and lysosomal GAL activities.

To confirm senescence, we performed staining for SA- $\beta$-GAL [16]. Our result showed that staining was more pronounced in stress-induced senescence than in non-stressed cells. However, we observed very strong correlations between SA- $\beta$-GAL staining and GAL activity on individual days of the experiment in both non-stressed and stressed fibroblast cell cultures. Moreover, our results prove that, based on the concentration of lysosomal GAL, one can specify the level 
A
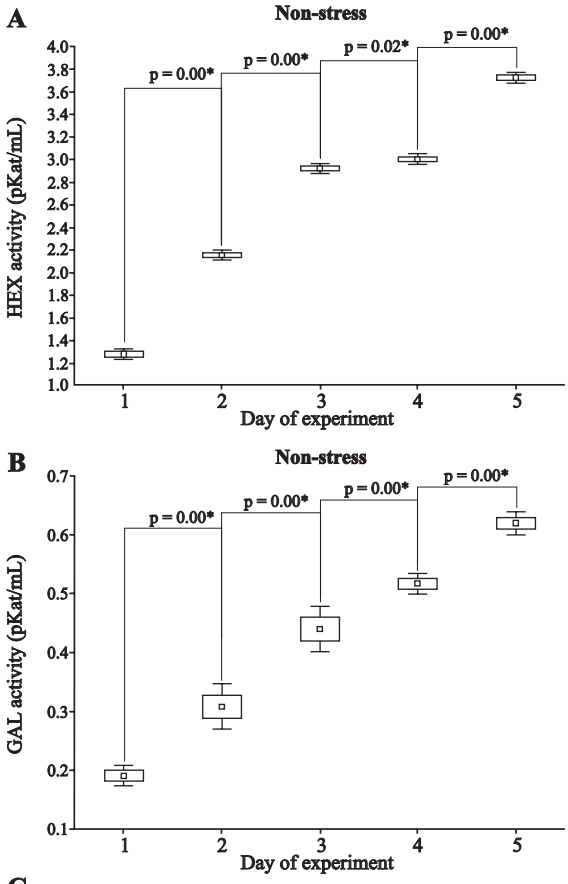

C

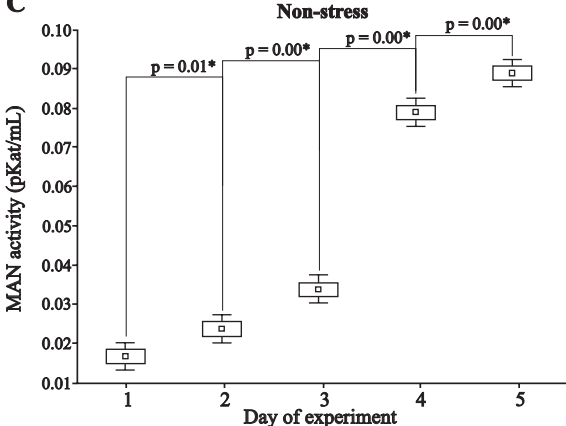

D

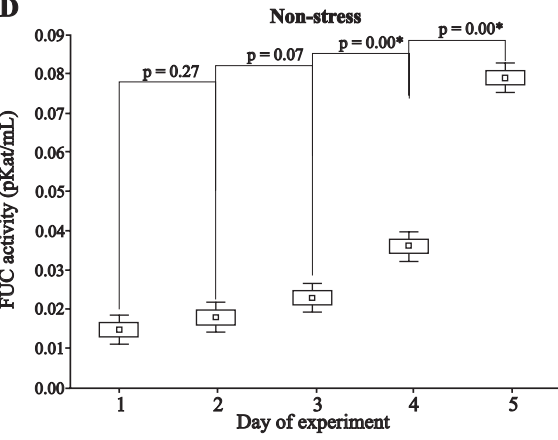

E

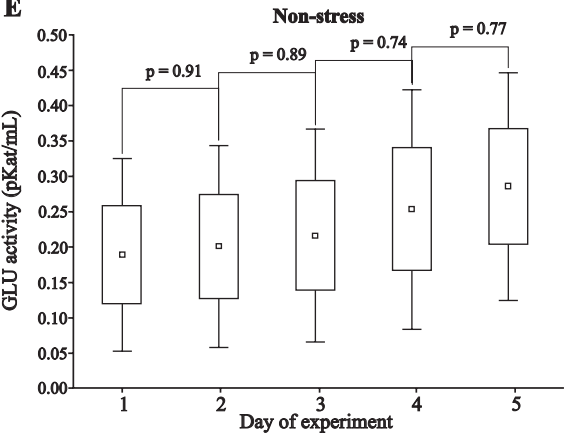

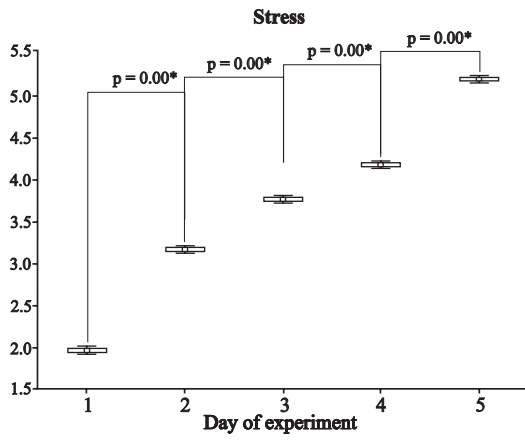

Stress

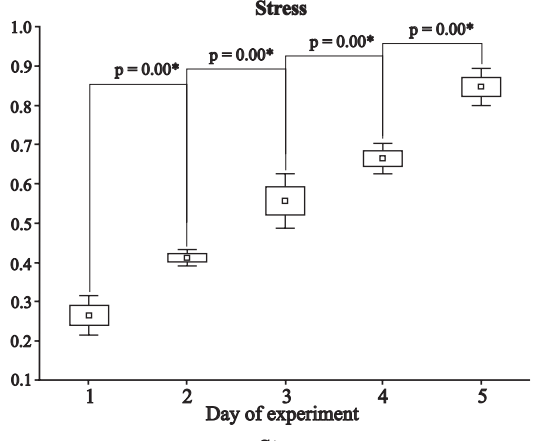

Stress
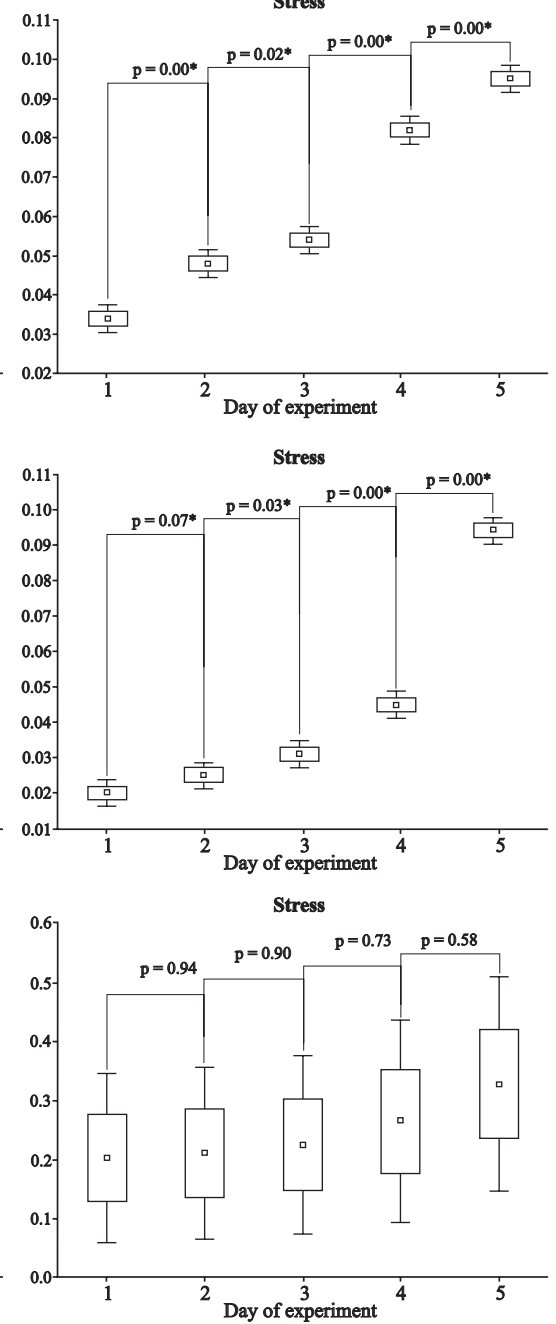

Figure 1. Changes of lysosomal exoglycosidases activity between individual days of experiment in non-stressed and stressed fibroblast cell cultures. Abbreviations: HEX $-\mathrm{N}$-acetyl $\beta$-hexosoaminidase; GAL $-\beta$ galactosidase; MAN $-\alpha$ mannosidase; FUC $-\alpha$ fucosidase; GLU $-\beta$ glucuronidase 
A

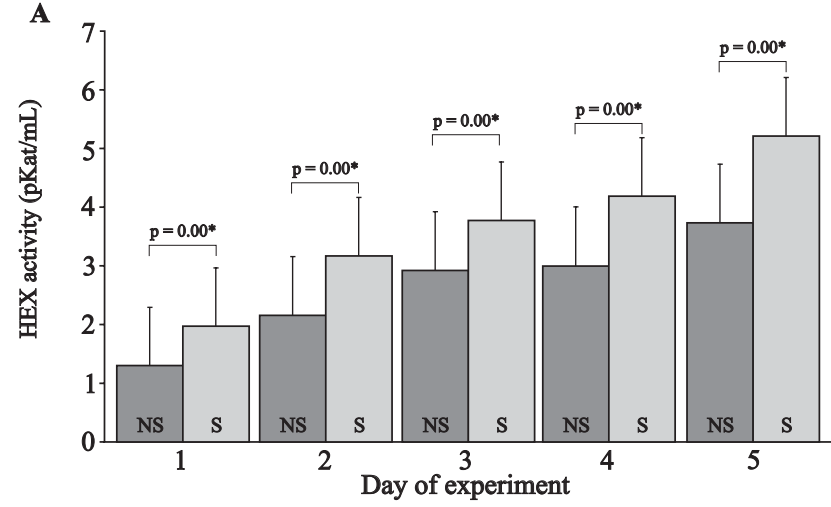

C

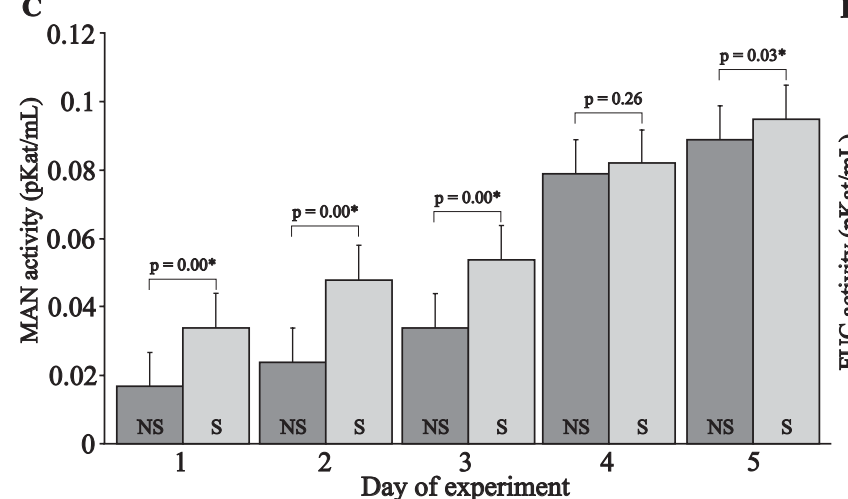

B

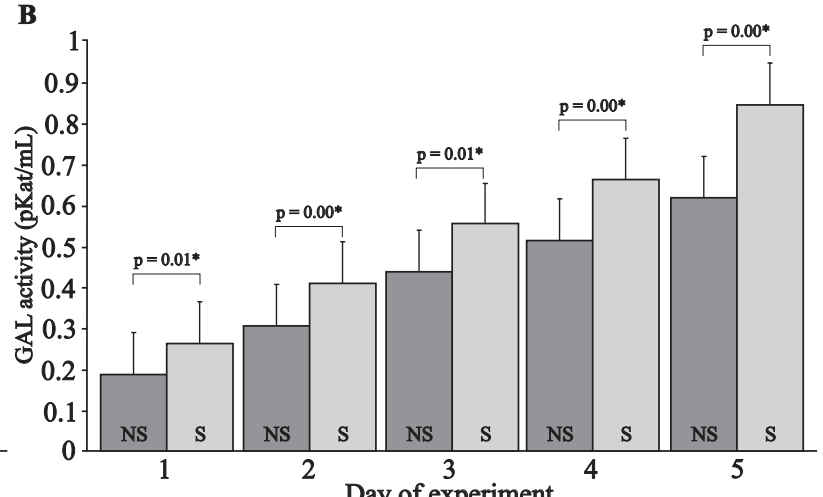

D

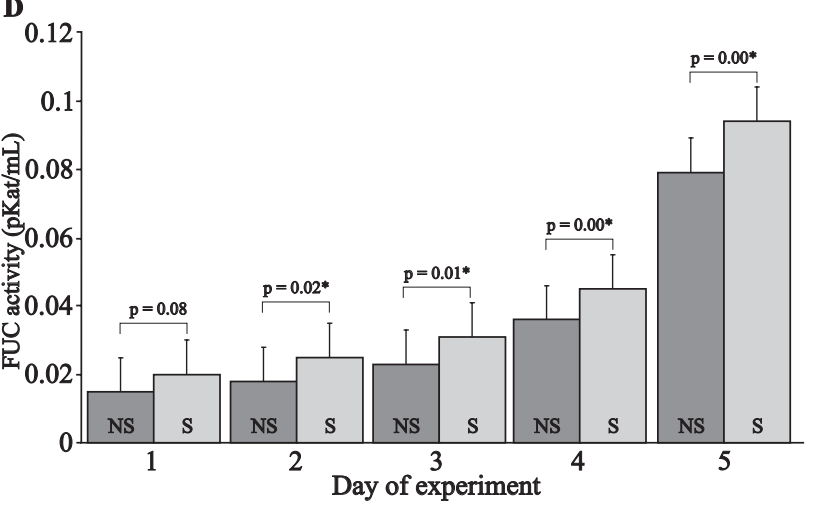

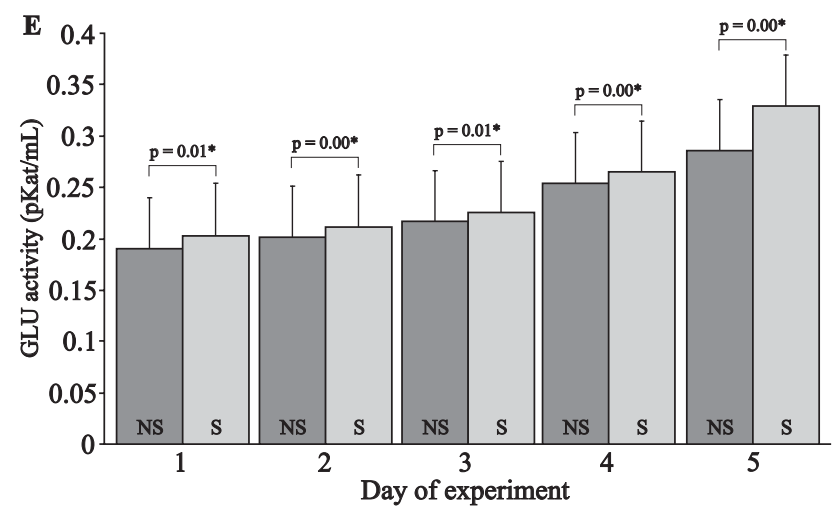

Figure 2. Changes of lysosomal exoglycosidases activity in each day of experiment, in the cell lysate of stressed fibroblasts, in comparison to the non-stressed cells. Abbreviations: HEX $-\mathrm{N}$-acetyl $\beta$-hexosoaminidase; GAL $-\beta$ galactosidase; MAN $-\alpha$ mannosidase; FUC $-\alpha$ fucosidase; GLU $-\beta$ glucuronidase; $\mathrm{S}-$ stress; NS - non-stress

of SA- $\beta$-GAL activity (Figure 4 ), that is the level of the cell culture ageing. SA- $\beta$-GAL activity that is cytochemically detected using 5-bromo-4-chloro-3-indolyl- $\beta$-D-galactoside (X-gal) is highly time-consuming (24 hours). The determination of GAL activity according to our method takes only one hour, and, as our results show, it can be useful in assessing the degree of ageing of the cell culture.

Lysosomal enzymes are easily release from exposed on oxidative stress lysosomes. It has been shown that oxidative stress causes an increased level of reactive oxygen species and that apoptotic cell death may be induced under severe oxidative stress [17].
Severe oxidative stress causes disruption of the lysosomes, and a release of a high concentration of lysosomal enzymes into the cytosol results in cell death. Less severe oxidative stress applied to the cells accelerates the ageing of cells and is the most important means of activating lysosomes, something which is observed as increased lysosomal activity [18].

Our results showed that on each day of the cell culture growth, there was a significant increase in the activity of all enzymes examined, with the exception of GLU, both in the cells which undergo replicative senescence and stress-induced senescence. As we expected, the activities of all examined exoglycosidases 

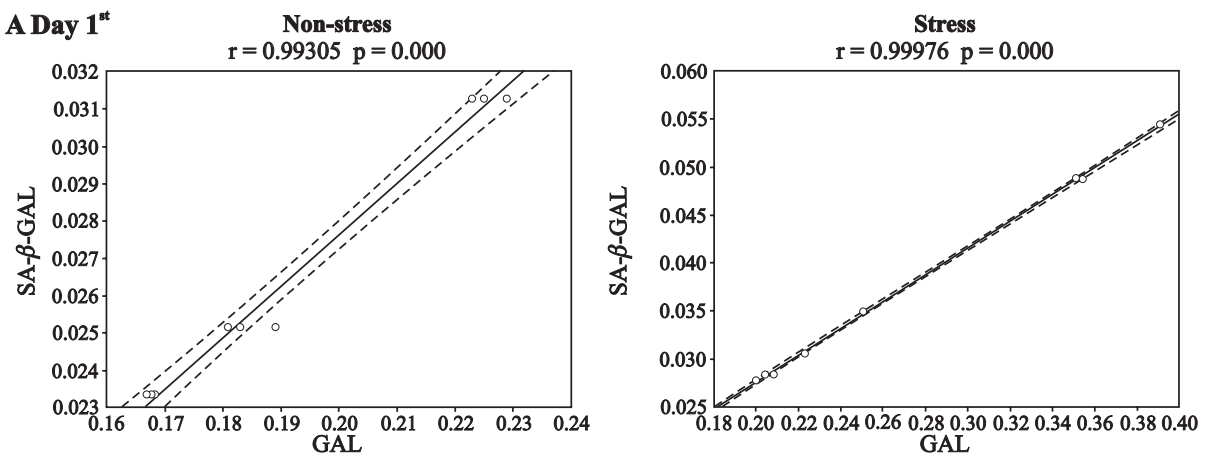

B Day $2^{\text {nd }}$

Non-stress
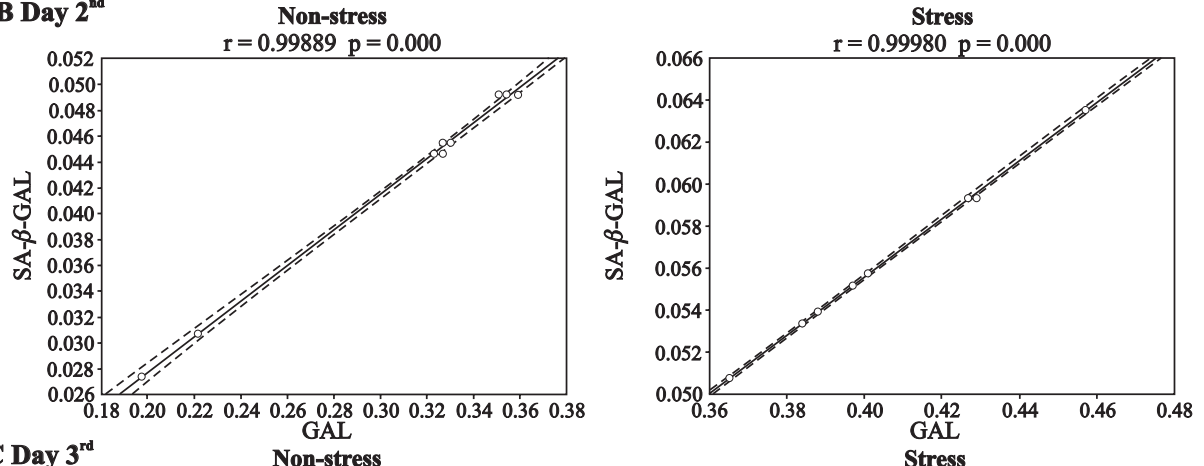

C Day $3^{\text {rd }}$

Non-stress
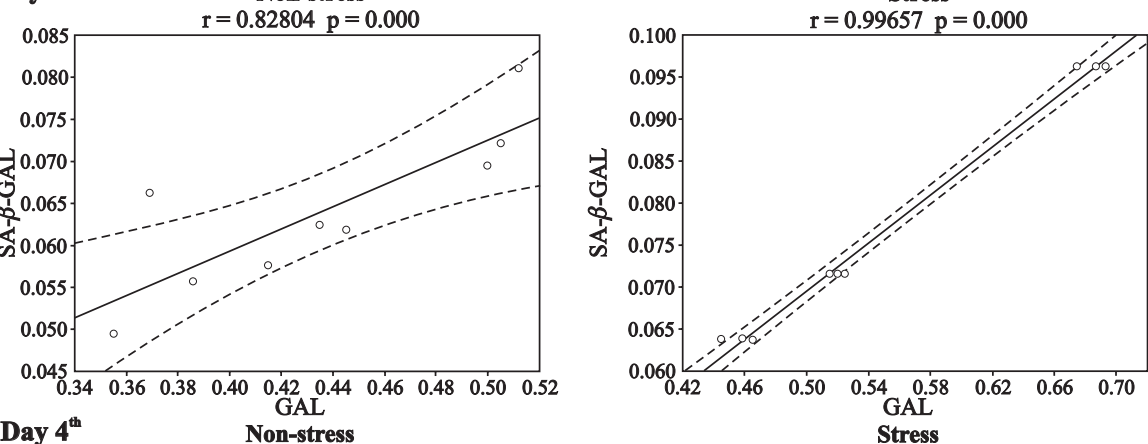

D Day $4^{\text {th }}$

Non-stress
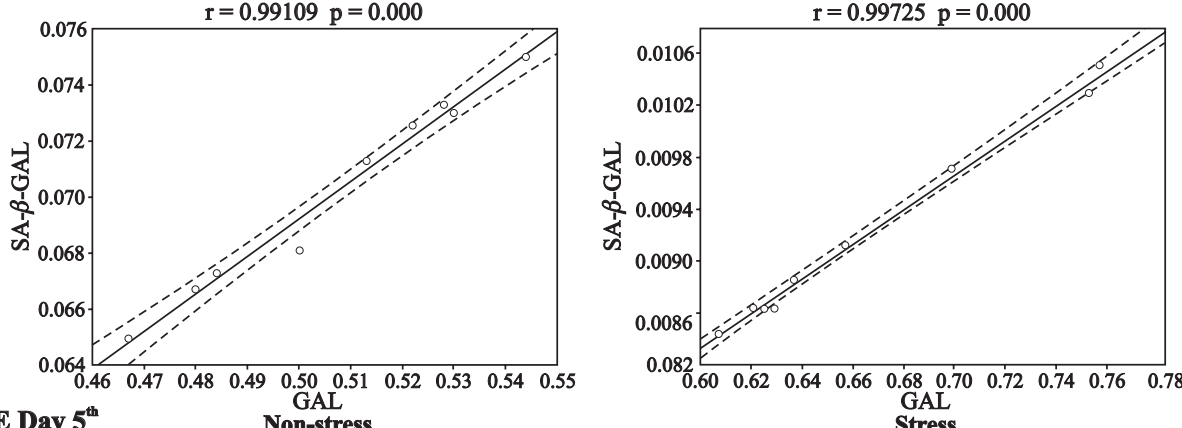

E Day $5^{\text {th }}$ Non-stress
$=0.99652 \mathrm{p}=0.000$
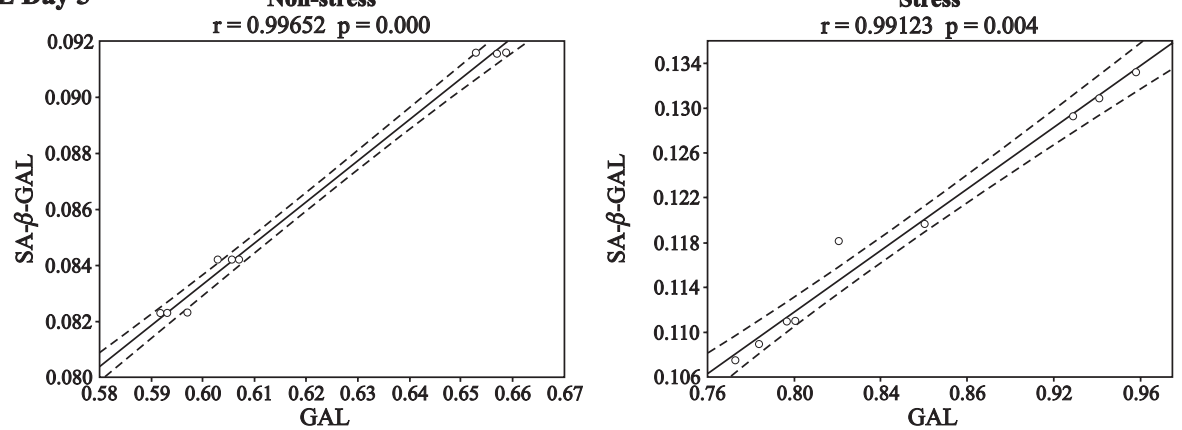

Figure 3. Correlations between SA- $\beta$-GAL staining and the GAL activity in individual days of experiment in non-stressed and stressed fibroblast cell culture. Abbreviations: GAL $-\beta$ galactosidase; SA $\beta$-GAL — senescence-associated $\beta$-galactosidase 
A

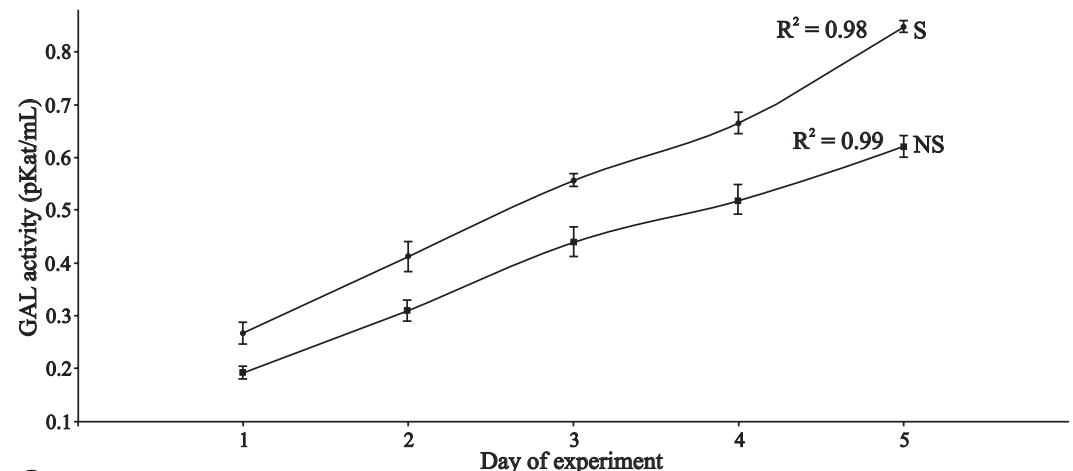

B

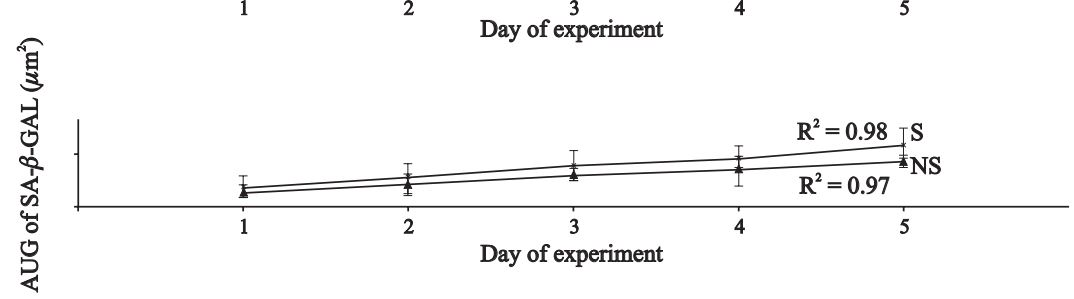

Figure 4. $2^{\text {nd }}$-order polynomial regression line of $\beta$ galactosidase (GAL) activities and senescence-associated $\beta$-galactosidase (SA- $\beta$-GAL) staining in individual days of experiment in non-stressed and stressed fibroblast cell culture. Abbreviations: AUC - area under curve; S — stress; NS — non-stress

were significantly higher in $t$-BHP treated cells compared to untreated cells. The results showed that the activities of lysosomal exoglycosidases increased with the intensity of the ageing of the cell culture. Oxidative stress is a factor that enhances ageing, resulting in a significant increase in exoglycosidase activity compared to the cells undergoing replicative senescence.

An increase in the activity of lysosomal exoglycosidases may demonstrate an intensification of degradative processes as a result of toxic substances [19$21]$, neoplasm [22, 23], inflammation or autoimmune disease [24-26]. Lysosomes are the main degradative sites in the cells, receiving material from phagosomes, endosomes and autophagosomes. Because the lysosomal enzymes are so abundant and so potent, their elevated release of exoglycosidases may be the reason for the cell damage through the breakdown of cellular components [27].

Replicative and stress-induced senescence results in drastic changes in the level of lysosomal exoglycosidases and results in enhanced lysosomal degradative capacity.

\section{References}

1. Hayflick L, Moorhead PS. The serial cultivation of human diploid cell strains. Exp Cell Res. 1961;25:585-621.

2. Hayflick L. The limited in vitro lifetime of human diploid cell strains. Exp Cell Res. 1965;37:614-636.

3. Chainiaux F, Magalhas J, Eliars F, Remacle J, Toussaint O. UVB-induced premature senescence of human diploid skin fibroblasts. Int J Biochem Cell Biol. 2002;34:1331-1339.

4. Stępień A, Izdebska M, Grzanka A. The types of cell death. Postępy Hig Med Dośw. 2007;61:420-428.
5. Dimiri GP, Lee X, Basile G et al. A biomarker that identifies senescent human cells in culture and in aging skin in vivo. Proc Natl Acad Sci USA. 1995;92:9363-9367.

6. Zwierz K, Zalewska A, Zoch-Zwierz W. Isoenzymes of N-acetyl-beta-hexosaminidase. Acta Biochim Polon. 1999; 46:739-757.

7. Cuervo AM. Autophagy: in sickness and in health. Trends Cell Biol. 2004;14:70-77.

8. Hilt W, Wolt DH. A field guide to ubiquitylation. Cell Mol Life Sci. 2004;61:1546-1561.

9. Zwierz K, Gindzieński A, Ostrowska L, Stankiewicz-Choroszczucha B. Metabolism of glycoconjugates in human gastric mucosa - a review. Acta Med Hung. 1989;46:275$-288$.

10. Edge CJ, Rademacher TW, Wormald MR et al. Fast sequencing of oligosaccharides. The reagent- array analysis method. Proc Natl Acad Sci. 1992;89:63338-66342.

11. Messer P, Christian R, Kolbe J, Schultz GS, Sleytr UB. Analysis of a novel linkage unit of O- linked carbohydrates from the crystalline surface layer glycoprotein of Clostridium thermohydrosulfuricum. J Bacteriol. 1992;174:2236-2240.

12. Chojnowska S, Kępka A, Szajda SD, Waszkiewicz N, Bierć M, Zwierz K. Exoglycosidase markers of diseases. Biochem Soc Trans. 2011;39:406-409.

13. Pavlica S, Gebhardt R. Protective effects of ellagic and chlorogenic acids against oxidative stress in PC12 cells. Free Rad Res. 2005;39:1377-1390.

14. Marciniak J, Zalewska A, Popko J, Zwierz K. Optimization of an enzymatic method for the determination of lysosomal $\mathrm{N}$-acetyl-beta-D-hexosoaminidase and beta-glucuronidase in synovial fluid. Clin Chem Lab Med. 2006;44:933-937.

15. Chatteriee S, Velicer LF, Sweeley CCJ. Glycosphingolipid Glycosyl Hydrolases and Glycosydases of synchronized Human KB Cells. J Biol Chem. 1975;250:4972-4979.

16. Dimri GP, Lee X, Basile $\mathrm{G}$ et al. A biomarker that identifies senescent human cells in culture and in aging skin in vivo. Proc Natl Acad Sci USA. 1995;92:9363-9367. 
17. Yoon J, Bang SH, Park J-S, Chang S-T, Kim Y-H, Min J. Increased in Vitro Lysosomal Function in Oxidative Stress-Induced Cell Lines. Appl Biochem Biotechnol. 2011;163:1002-1011.

18. Yoon J, Park J-M, Jung S-K, Kim K-Y, Min J. Characterization of antimicrobial activity of the lysosomes isolated form Saccharomyces cerevisiae. Curr Microbiol. 2009;59:48-52.

19. Waszkiewicz N, Szajda SD, Jankowska A et al. The effect of the binge drinking session on the activity of salivary, serum, urinary beta Hexosaminidase: Preliminary data. Alcohol Alcohol. 2008;43:446-450.

20. Waszkiewicz N, Szajda SD, Jankowska A et al. Catabolism of salivary glycoconjugates in acute ethanol intoxication. Med Sci Monit. 2009;15:413-417.

21. Knaś M, Karaszewska K, Szajda SD, Zarzycki W, Dudzik D, Zwierz K. Saliva of patients with Type I diabetes: effect of smoking on activity of lysosomal exoglycosidases. Oral Dis. 2006;12:278-282.

22. Szajda SD, Jankowska A, Zwierz K. Carbohydrate markers in colon carcinoma. Dis Markers. 2008;25:233-242.
23. Bierć M, Minarowski Ł, Woźniak $€$ et al. The activity of selected glycosidases in salivary gland tumors. Folia Histochem Cytobiol. 2010;48:471-474.

24. Popko J, Marciniak J, Ilendo E et al. Profile of exoglycosidases in synovial cell cultures derived from human synovial membrane. Cell Biochem Biophys. 2008;51:89-95.

25. Popko J, Marciniak J, Zalewska A et al. Activity of N-acetylbeta-hexosoaminidase and its isoenzymes in serum and synovial fluid from patients with different arthropathies. Clin Exp Rheumatol. 2006;24:690-693.

26. Popko J, Zalewska A, Gołaszewska Z et al. Comparative analysis of hexosoaminidase and cathepsin $\mathrm{D}$ expression in synovial fluid of patients with rheumatoid arthritis and traumatized joints. Clin Exp Rheumatol. 2005;23:725$-726$.

27. Lee S-J, Koh J-Y. Roles of zinc and metallothionein-3 in oxidative stress-induced lysosomal dysfunction, cell death, and autophagy in neurons and astrocytes. Molecular Brain. 2010;3:30.

Submitted: 10 November, 2011

Accepted after reviews: 24 January, 2012 